\title{
El ámbito de la evaluación económica de intervenciones de salud
}

\author{
JORGE JIMÉNEZ DE LA J. ${ }^{1, a}$, GABRIEL BASTÍAS S. ${ }^{1, b}$
}

\section{The scope of economic evaluation of healthcare interventions}

The World Health Organization (WHO) has contributed to the conceptualization of a Health System stating that it has three main objectives and four functions. The main objective is Health Status, measured in Disability Adjusted Life Expectancy (DALE).The others two are Responsiveness to the people's Non-Medical Expectations and Equity in Financing. This last is a specific indicator of economic nature. Economics has an increasing role in the health systems and the recognition of this component by actors in health services is more and more important every day. Medical professionals need to understand its meaning and relevance in many senses of medical care. Economic evaluations, mainly cost-effectiveness of health programs and socio-sanitary interventions are more complex to carry out than common social evaluations, whose context is more restricted. When formulating modern health policies, the declared objective is always the improvement and quality of healthcare. Nevertheless, in recent years policy makers have insisted in the need to secure economic rationality of interventions, both inside the program, thus achieving internal technical efficiency, as towards the whole society achieving allocative efficiency. When the purpose is to evaluate health programs, economic evaluation integrates costs with effectiveness and considers the epidemiologic profile and social preferences. The priority setting included in the Health Guarantees Plan (AUGE or GES) established by the Chilean Ministry of Health in 2005 is a clear example of a process of Health Technology Assessment, where cost-effectiveness studies were used to set the program and establish priorities. This is the first in a series of papers related to economic evaluation of healthcare interventions. This paper aims to contribute to the development of this field, providing basic concepts and its main applications to health care in Chile.

(Rev Med Chile 2010; 138 (Supl 2): 71-75).

Key words: Priority setting, health economics, economics evaluation, Chile.

\section{RESUMEN}

La Organización Mundial de la Salud (OMS) ha contribuido a la conceptualización de lo que es un sistema de salud, en el cual integra tres objetivos y cuatro funciones. El objetivo principal es la salud, la cual es medida con el indicador Expectativa de Vida Ajustada por Discapacidad (EVAD), los otros dos son Capacidad de Respuesta a las Expectativas No Medicas y la Equidad o Justicia Financiera. Esta última es una condición explícita de carácter económico. La economía tiene un papel creciente en los sistemas de salud y es importante que los actores principales del sistema, los profesionales médicos, estén al tanto de ello y logren comprender su dimensión. Las evaluaciones económicas, principalmente las de costo-efectividad de los programas de

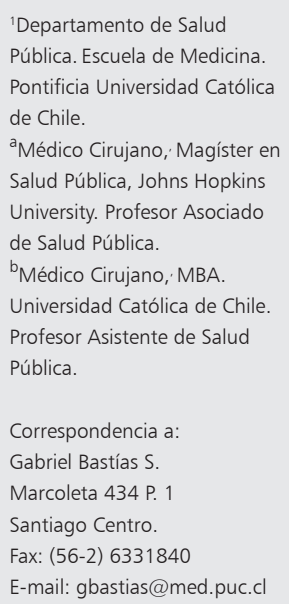


salud e intervenciones socio-sanitarias siguen siendo más complejas de realizar que las evaluaciones en otras áreas sociales, cuyo contexto es más restringido o específico. En la formulación de políticas de salud modernas, el objetivo declarado es siempre el de mejorar la calidad de los cuidados y de la atención médica. Sin embargo, ahora se hace insistiendo en que uno de los componentes esenciales de la calidad es la racionalidad económica de las intervenciones, tanto internas al propio programa, logrando eficiencia técnica, como al conjunto de la sociedad logrando eficiencia distributiva. Cuando se trata de evaluar Tecnologías Sanitarias, uno de sus componentes es la evaluación económica que integra costos con efectividad clínica y considera el perfil epidemiológico y las preferencias sociales. Estás últimas se pueden incorporar a través de la valoración de los estados de salud (en "outcomes" como Quality-Adjusted LifeYears-QALY) y en el uso de una tasa de descuento social. La determinación de las condiciones de salud incluidas en el Plan de Garantías en Salud (AUGE o GES) es un ejemplo de un proceso de Evaluación de Tecnologías Sanitarias (ETESA). En dicho proceso se hizo una aproximación a incluir explícitamente la evaluación económica, a través de los estudios de costo-efectividad, en la priorización para determinar las condiciones de salud que se integrarían al plan. Este es el primero de una serie de artículos sobre el ámbito de la evaluación económica de intervenciones de salud la que pretende entregar a la comunidad médica un conjunto de contenidos que dan luces sobre el desarrollo de la disciplina y sus conceptos principales, a la vez que sus aplicaciones posibles a las actividades de la salud en nuestro país.

Palabras clave: Determinación de prioridades, economía de la salud, evaluación económica, Chile.

$\tau$ a Organización Mundial de la Salud (OMS) ha conceptualizado un sistema de salud, en el que se integran tres objetivos y cuatro funciones. El objetivo principal es la salud, medida con el indicador Expectativa de Vida Ajustada por Discapacidad (EVAD), los otros dos objetivos son Capacidad de Respuesta a las Expectativas No Medicas y la Equidad o Justicia Financiera. Esta última es una condición explícita de carácter económico y de equidad. En las cuatro funciones de un sistema de salud: Rectoría, Generación de Recursos, Finanzas y Provisión de Servicios, se dan aspectos económicos muy claros como el financiamiento de la generación de recursos tecnológicos, de infraestructura y de personal calificado entre otros. En la provisión de servicios de salud el aspecto económico es bastante nítido ${ }^{1}$ y es precisamente en esta área donde la disciplina de la evaluación económica tiene más aplicación.

La economía, entendida como la ciencia del uso correcto y efectivo de los recursos disponibles, tiene un papel creciente en los sistemas de salud y es importante que los actores principales del sistema, los profesionales médicos, estén al tanto de ello y logren comprender su dimensión.

Existen espacios de la función social salud en los cuales la evaluación social y económica es cada día más relevante. Por de pronto, las tecnologías médicas, ya sean de diagnóstico o terapéuticas en sus variadas formas. Las intervenciones agregadas en forma de programas de salud, con componentes médico-sociales, tanto para individuos como a nivel poblacional o comunitario, también son objeto de evaluaciones económicas, aunque su evaluación es de mayor dificultad por tratarse de intervenciones complejas.

\section{Consideraciones económicas en la toma de decisiones en salud}

A niveles macro políticos, las consideraciones económicas sobre el financiamiento de las intervenciones son cada vez más frecuentes y utilizadas como un argumento para la toma de decisiones. Así ha ocurrido en Chile desde 1997, cuando la Dirección de Presupuestos del Ministerio de Hacienda inició su Programa de Evaluación de políticas y programas ${ }^{2}$. Inicialmente las evaluaciones fueron realizadas con el método del Marco Lógico, posteriormente en 2001 se inició la Evaluación de Impacto y posteriormente la Evaluación 
Comprehensiva del Gasto en una aproximación más econométrica. Han sido evaluados numerosos programas del área salud en este proceso, entre otros los de inmunizaciones, prevención de VIH/SIDA, el subsidio maternal, el de alimentación complementaria en el conjunto de las intervenciones propiamente sanitarias. También se ha evaluado programas de apoyo financiero a los usuarios como el de préstamos médicos de FONASA o el de las prestaciones complejas de la misma organización.

Las evaluaciones económicas de salud, las que en términos prácticos, se definen como un análisis comparativo de cursos alternativos de acción en términos de sus costos y consecuencias en salud ${ }^{3}$, principalmente las de costo-efectividad de los programas de salud e intervenciones socio-sanitarias, siguen siendo más complejas de realizar que las evaluaciones en otras áreas sociales, cuyo contexto es más restringido o específico. Una de las primeras aplicaciones de esta metodología es conocida como fármaco-economía, que realiza estudios sobre el uso y acceso a los medicamentos y sus consecuencias en el manejo de las enfermedades.

Para Weatherly y colaboradores ${ }^{4}$ los cuatro principales desafíos de las evaluaciones económicas de los programas de salud pública son:

- La atribución de los efectos

- La medición y valoración de los resultados en indicadores

- La identificación de los costos y consecuencias intersectoriales, $y$

- Las consideraciones de equidad

Efectivamente, cada vez que se inicia un estudio de evaluación económica de un programa de salud relativamente amplio, los investigadores se enfrentan a un esfuerzo mayor. Esto ocurre porque se debe integrar no sólo variables propias de salud sino también aquellas atribuibles al desarrollo socio-económico en general ya que los aportes o los impactos de otros sectores, tales como educación, vivienda y medio-ambiente pueden ser muy influyentes en salud. Así surgen necesidades de mayor precisión a objeto de tener conclusiones que sean verdaderamente robustas para orientar las políticas.

Al nivel de las prestaciones médicas individuales que constituyen los llamados "Bienes Privados", la aplicación de métodos de evaluación económica es más antigua y reconocida. Así la Fármaco- economía, disciplina establecida y relativamente madura, en sus orígenes fue un término usado como sinónimo de evaluación económica ${ }^{5}$. No obstante la Evaluación Económica, en su sentido más amplio, ha sido una disciplina donde se pueden evaluar intervenciones comunitarias, tratamientos médico, uso de medicamentos, formas de entregar las prestaciones, etc.

\section{Evaluaciones económicas previas a la introducción de nuevas tecnologías}

La intervención sanitaria más calificada por su reconocida eficacia, efectividad y costo-efectividad es la de las vacunas. Varias enfermedades han logrado ser controladas y erradicadas por el uso de vacunas. La costo-efectividad de ellas ha sido ponderada infinitas veces y los partidarios de expandir el uso de las vacunas así lo hacen saber ${ }^{6}$. Normalmente las vacunas de mayor costo-efectividad son aquellas que combaten enfermedades de antígeno único, como la poliomielitis y el sarampión, cuyo costo por niño inmunizado es de menos de un dólar. Diferente es el caso de aquellas enfermedades que tienen varios antígenos, como las contra el neumococo, cuyo costo sube cincuenta o cien veces. Mucho más complejo aún es el caso de vacunas asociadas a una infección relacionada a un cáncer, como la del Virus del Papiloma Humano, cuyo costo es bastante mayor y su efectividad (aun no completamente probada) se observa a quince o veinte años plazo $^{7-9}$.

La evaluación de tecnologías sanitarias (ETESA) ha pasado a ser una verdadera institución en los sistemas de salud bien establecidos. Esta ha sido definida por la Office of Technology Assessment (OTA), creada por el Congreso de los Estados Unidos en 1972, como una "forma de investigación que examina las consecuencias técnicas, sociales, económicas, éticas y legales derivadas del uso de la tecnología, que se producen a corto y largo plazo y que pueden ser tanto directas como indirectas y tanto sobre los efectos deseados como sobre los indeseados".

Las experiencias europeas y americanas de esta línea de apoyo a las políticas de inversión y compra de innovaciones en salud está siendo liderada por el Nacional Institute for Health and Clinical Excellence (NICE) del Servicio Nacional de Salud del Reino Unido. Su aporte al mejoramiento de la 
calidad de la atención, basado en la confección de protocolos y guías clínicas, las revisiones permanentes de la evidencia en la literatura científica y la capacitación para el mejoramiento de la calidad son proverbiales ${ }^{10}$. Muchos países buscan imitar esta experiencia, entre otros, el Gobierno norteamericano de Barak Obama en su proyecto de reforma de salud de $2009^{11}$. En el proyecto inicial, discutido en el congreso, se incluye la creación de una agencia que cumpla una tarea semejante a la británica.

En la formulación de políticas de salud modernas, el objetivo declarado es siempre el de mejorar la calidad de los cuidados y de la atención médica. Sin embargo, ahora se hace insistiendo en que uno de los componentes esenciales de la calidad es la racionalidad económica de las intervenciones, tanto internas al propio programa, logrando eficiencia técnica, como al conjunto de la sociedad logrando eficiencia distributiva.

\section{Evaluaciones económicas de impacto}

Otra de las formas de mirar la evaluación de las intervenciones es medir su impacto en indicadores definidos y aceptados. Clásicamente se ha utilizado la tasa de mortalidad infantil para medir el nivel de desarrollo de una sociedad. Un alto número de niños fallecidos antes de cumplir el primer año de vida será un indicador de subdesarrollo, mientras que las bajas tasas serán homologadas a un buen o alto desarrollo socioeconómico. Más recientemente, basado en la Teoría de Capital Humano, se busca medir en términos monetarios el valor económico de las muertes evitadas, especialmente aquellas que ocurren prematuramente, como las infantiles o de adultos jóvenes. Atribuir un valor monetario a la vida (o al evitar una muerte) es un ejercicio que parecía algo macabro, pero que finalmente puede poner en perspectiva comparable dos o más programas que compiten por los mismos recursos. Así será muy claro para la autoridad sanitaria o política si evitar una muerte por sarampión es más beneficioso (en función de sus costos) que evitar una muerte con un trasplante de hígado. Mientras el valor de una vacuna es un dólar, (donde un programa de miles de vacunas se evitarán miles de muertes), un trasplante de hígado puede costar diez mil dólares y beneficiaría sólo a una persona. La decisión priorizada, desde una perspectiva económica será favorable a la primera. Sin dudas, técnica y éticamente, esa decisión debe ser establecida.

Para evitar medir los beneficios de la intervenciones en salud en términos monetarios los investigadores han desarrollados medidas genéricas en salud como los Disability-Adjusted LifeYears (DALY) y los Quality-Adjusted Life-Years (QALY) donde las intervenciones que maximizan los QALY (y minimizan los DALY) son aquellas que se priorizan.

\section{Integración de la evaluación económica con los aspectos socio-epidemiológicos y de preferencias sociales}

Cuando se trata de evaluar programas de salud, el componente de evaluación económica, que integra costos y efectividad clínica, deberá además integrar lo epidemiológico y las preferencias sociales. El conocido algoritmo para determinar las condiciones de salud que se verían incorporadas al plan de Garantías en Salud (AUGE o GES) ${ }^{12}$ fue la primera vez en Chile en se que explicitó públicamente la secuencia del razonamiento sanitario con sentido epidemiológico, carga de enfermedad, efectividad y costo de las intervenciones y de las preferencias sociales de la comunidad. El primer requisito para incluir una condición de salud es su impacto en la carga de enfermedad, el segundo es el de la efectividad y disponibilidad de medios diagnósticos y terapéuticos cruzados con su costo económico, la costo-efectividad. Las preferencias sociales, que dicho en palabras simples, constituye el juicio social de la comunidad de la precedencia de los problemas y las soluciones. Este paso, muchas veces el más controversial, no es tan fácil de aplicar ni de medir, ya que muchos grupos de presión, favorables a resolver un problema que les es cercano, se movilizan con mayor eficacia que los sufrientes de otro problema menos visible o más olvidado. En estas controversias, el argumento de tipo económico, si bien no definitivo, podrá ser necesario y muy útil para cerrar debates y tomar decisiones compartidas social y políticamente.

\section{Conclusiones}

Este es el primero de una serie de artículos sobre el ámbito de la evaluación económica de 
intervenciones de salud la que pretende entregar a la comunidad médica un conjunto de contenidos que dan luces sobre el desarrollo de la disciplina, sus conceptos principales y sus aplicaciones posibles a las actividades de la salud en nuestro país.

\section{Referencias}

1. Organización Mundial de la Salud (OMS). Informe sobre la Salud en el Mundo 2000. Mejorar el desempeño de los Sistemas de Salud. Capítulo 2: 49-50. OMS Ginebra.

2. Gobierno de Chile. Ministerio de Hacienda. Dirección de Presupuesto (DIPRES). Evaluación de Programas e Instituciones. Disponible en: www.dipres.cl/572/propertyvalue-15697.html [Consultado el 19 de noviembre de 2009].

3. Drummond MF, O'brien B, Stoddart GL, Torrance GW. Methods for the economic evaluation of health care programs. $2^{\circ}$ edición ed. Oxford: Oxford University Press; 1997

4. Weatherly H, Drummond M, Claxton K, Cookson R. Ferguson B, Godfrey C, et al. Methods for assessing the cost-effectiveness of public health interventions: Key challenges and recommendations. Health policy 2009; 93: 85-92.
5. Aslam A. Evidence-Based Decision Making: Using Submission Guidelines to Inform Formulary Approvals. The American Journal of Managed Care, Editorial 1999; 5: 356-358

6. Bloom D, Canning D, Weston M. The Value of Vaccination. World Economics 2005; 6: 15-40.

7. Bosch FX, Burchell AN, Schiffman M, Giuliano AR, De Sanjose S, Bruni L, et al. Epidemiology and natural history of human papillomavirus infections and typespecific implications in cervical neoplasia. Vaccine 2008; (Suppl 10): K1-K16.

8. Bosch FX, Lorincz A, Muñoz N, Meijer CJ, Shah KV. The causal relation between human papillomavirus and cervical cancer. J Clin Pathol 2002; 55: 244-65.

9. Bosch FX, De Sanjose S. Human papillomavirus in cervical cancer. Curr Oncol Rep 2002; 2: 175-83.

10. NHS. National Institute for Health and Clinical Excellence (NICE). NHS Evidence. Disponible en: www.nice. org.uk/nhsevidence/ [Consultado el 19 de noviembre de 2009].

11. Kerr DJ, Scott M. British lessons on health care reform. N Engl J Med 2009; 361: e21(1)-(3).

12. Bastías G, Pantoja T, Leisewitz T, Zarate V. Reforming health care in Chile: current and future challenges. Can Med Assoc J 2008; 179: 1289-92. 